\title{
Effects of calcium and chitosan treatments on controlling anthracnose and postharvest quality of papaya (Carica papaya L.)
}

\begin{abstract}
This study was conducted to evaluate the in vitro fungicidal effects of calcium and chitosan on Colletotrichum gloeosporioides and to as well determine their effects on storage life and quality of papaya. Potato Dextrose Agar (PDA) incorporated with calcium at different concentrations $(1.5,2.5$ or $3.5 \%)$ or in combination with chitosan at $0.75 \%$ or chitosan alone were used as treatments for in vitro tests. Uncorporated treatments with PDA and untreated fruits as control used on papaya fruits for storage life and quality evolutions. Chitosan had the greatest effect against Colletotrichum gloeosporioides in both in vitro and in disease incidence $(\%)$ on papaya fruits compared to calcium treatment and as well as control. Calcium reduced spores germination significantly as calcium concentrations increased from 2.5 to $3.5 \%$, compared to the $1.5 \%$ and control treatments. However, it did not show any fungicidal effects on mycelial growth. The combination of $2.5 \%$ calcium with chitosan $0.75 \%$ completely inhibited spore germinations and significantly inhibited mycelia growth compared to calcium individual treatments and as well as control. Anthracnose disease incidence (\%) was significantly controlled (5.6\%) using calcium at $2.5 \%$ combined with chitosan compared with the other treatments. This demonstrated the best effect on controlling anthracnose disease incidence for papaya fruits. Moreover, this treatment proved able to extend the storage life of papaya fruits up to 33 days of storage life while maintaining valuable attributes of quality.
\end{abstract}

Keyword: papaya, chitosan, calcium, papaya quality, Colletotrichum gloeosporioides 